\title{
RETENÇÃO DE PESO PÓS-PARTO EM \\ MULHERES ASSISTIDAS NO SERVIÇO \\ PÚBLICO DE SAÚDE: ESTUDO DE COORTE
}

\author{
POSTPARTUM WEIGHT RETENTION IN \\ WOMEN ASSISTED IN THE PUBLIC HEALTH \\ SERVICE: COHORT STUDY
}

\section{RETENCIÓN DE PESO POSPARTO EN MUJERES ASISTIDAS EN EL SERVICIO DE SALUD PÚBLICA: ESTUDIO DE COHORTE}

\author{
Lorenna Viccentine Coutinho Monteschio ${ }^{1}$ \\ Sonia Silva Marcon ${ }^{2}$ \\ Evelin Matilde Arcain Nass ${ }^{3}$ \\ Cátia Campaner Ferrari Bernardy ${ }^{4}$ \\ Áurea Christina de Paula Corrêa ${ }^{5}$ \\ Patrícia Chatalov Ferreira ${ }^{6}$ \\ Débora Cristina Arruda ${ }^{7}$
}

Como citar este artigo: Monteschio LVC, Marcon SS, Nass EMA, Bernardy CCF, Corrêa ACP, Ferreira PC, et al. Retenção de peso pós-parto em mulheres assistidas no serviço público de saúde: estudo de coorte. Rev baiana de enferm. 2021;35:e43026.

\begin{abstract}
Objetivo: analisar a retenção de peso pós-parto em mulheres assistidas no serviço público de saúde em um município do Sul do Brasil. Método: estudo de coorte realizado com 85 puérperas. Dados socioeconômicos, obstétricos, antropométricos, hábitos alimentares, atividade física, amamentação e fatores emocionais foram coletados mediante entrevista em dois momentos: no hospital, no puerpério imediato; e no domicílio, seis meses após o parto. Na análise, utilizou-se estatística descritiva e inferencial. Resultados: a incidência da retenção de peso pós-parto maior que $1 \mathrm{~kg}$ foi de 54,1\%, associada ao ganho de peso gestacional excessivo (68,4\%), estado nutricional eutrófico/baixo peso no início da gestação $(65,8 \%)$ e excesso de peso seis meses pós-parto $(61,8 \%)$. Mulheres que não amamentaram exclusivamente até seis meses retiveram mais peso. A prevalência de insatisfação corporal foi alta $(82,4 \%)$. Conclusão: os fatores de risco para retenção de peso pós-parto foram estado nutricional eutrófico pré-gestacional e ganho de peso excessivo na gestação.
\end{abstract}

Descritores: Período Pós-parto. Ganho de Peso na Gestação. Alterações do Peso Corporal. Saúde da Mulher. Sistema Único de Saúde. Enfermagem.

\footnotetext{
Enfermeira. Doutora em Enfermagem. Coordenadora da Divisão de Internamento do Hospital Universitário de Maringá. Maringá, Paraná, Brasil. helorenn@gmail.com. http://orcid.org/0000-0002-1486-6898.

Enfermeira. Doutora em Filosofia da Enfermagem. Docente da Universidade Estadual de Maringá. Maringá, Paraná, Brasil. soniasilva.marcon@gmail.com. http://orcid.org/0000-0002-6607-362X.

Enfermeira. Doutora em Enfermagem. Secretaria Municipal de Saúde de Sarandi. Maringá, Paraná, Brasil. http://orcid.org/0000-0002-5। 40-3 I04

Enfermeira. Doutora em Ciências da Saúde. Docente da Universidade Estadual de Londrina. Londrina, Paraná, Brasil. http://orcid.org/0000-000 I-9723-1857.

Enfermeira. Doutora em Enfermagem. Docente da Universidade Federal de Mato Grosso. Cuiabá, Mato Grosso, Brasil. http://orcid.org/0000-0003-2091-6879.

Enfermeira. Universidade Estadual de Maringá. Maringá, Paraná, Brasil. http://orcid.org/0000-000 I-9409-5888.

Enfermeira. Mestre em Enfermagem. Hospital Universitário de Maringá, Universidade Estadual de Maringá. Maringá, Paraná, Brasil. http://orcid.org/0000-00032926-0300.
} 
Objective: to analyze postpartum weight retention in women assisted in the public health service in a municipality in southern Brazil. Method: cohort study conducted with 85 puerperal women. Socioeconomic, obstetric, anthropometric data, eating habits, physical activity, breastfeeding and emotional factors were collected through interviews in two moments: in the hospital, in the immediate puerperium; and at home, six months after delivery. Descriptive and inferential statistics were used in the analysis. Results: the incidence of postpartum weight retention greater than $1 \mathrm{~kg}$ was 54.1\%, associated with excessive gestational weight gain (68.4\%), eutrophic nutritional status/low weight at the beginning of pregnancy (65.8\%) and overweight six months postpartum (61.8\%). Women who did not breastfeed exclusively up to six months retained more weight. The prevalence of body dissatisfaction was high (82.4\%). Conclusion: the risk factors for postpartum weight retention were pre-gestational eutrophic nutritional status and excessive weight gain during pregnancy.

Descriptors: Postpartum Period. Gestational Weight Gain. Body Weight Changes. Women's Health. Unified Health System. Nursing.

Objetivo: analizar la retención de peso posparto en mujeres atendidas en el servicio público de salud en un municipio del sur de Brasil. Método: estudio de cohorte realizado con 85 puérperas. Los datos socioeconómicos, obstétricos, antropométricos, hábitos alimenticios, actividad física, lactancia materna y factores emocionales fueron recolectados a través de entrevistas en dos momentos: en el hospital, en el puerperio inmediato; y en casa, seis meses después del parto. En el análisis se utilizó estadística descriptiva e inferencial. Resultados: la incidencia de retención de peso posparto mayor de $1 \mathrm{~kg}$ fue de 54,1\%, asociada a aumento excesivo de peso gestacional (68,4\%), estado nutricional eutrófico/bajo peso al inicio del embarazo $(65,8 \%)$ y sobrepeso seis meses postparto (61,8\%). Las mujeres que no amamantaron exclusivamente hasta seis meses retuvieron más peso. La prevalencia de insatisfacción corporal fue alta (82,4\%). Conclusión: los factores de riesgo para la retención de peso posparto fueron el estado nutricional eutrófico pre-gestacional y el aumento de peso excesivo durante el embarazo.

Descriptores: Periodo Posparto. Ganancia de Peso Gestacional. Cambios en el Peso Corporal. Salud de la Mujer. Sistema Único de Salud. Enfermería.

\section{Introdução}

A retenção de peso pós-parto (RPPP) refere-se ao peso adquirido durante a gestação e que se mantém após o parto. Constitui importante fator de risco para o desenvolvimento de obesidade em mulheres ${ }^{(1)}$. O excesso de peso materno aumenta as chances de ocorrência da síndrome metabólica e do risco transgeracional da obesidade materno-infantil ${ }^{(2)}$.

Não há consenso na literatura sobre o ponto de corte ideal para categorização da RPPP, ficando muito na dependência do tempo de seguimento. Revisão sistemática, com 20 estudos publicados entre 2000 e 2013, encontrou a variação de 19,2\% de RPPP maior que $7,5 \mathrm{~kg}$ aos nove meses, e até 61,3\% de RPPP maior que $1 \mathrm{~kg}$ aos seis meses após o parto ${ }^{(3)}$.

O aumento excessivo de peso durante a gestação é o principal preditor da $\mathrm{RPPP}^{(1)}$, porém a influência de fatores socioeconômicos e de outros, como história obstétrica, amamentação, estresse, aspectos psicológicos e estilo de vida, ainda é investigada em busca de se compreender melhor o fenômeno ${ }^{(1,4)}$.
O ganho de peso gestacional (GPG) já é esperado tanto pelo crescimento fetal como pelo aumento das estruturas maternas. Durante o pré-natal, é recomendado o monitoramento do peso, com verificação em todas as consultas ${ }^{(5)}$. Entretanto, estudo realizado no âmbito da $15^{\underline{a}}$ regional de saúde do estado do Paraná, por exemplo, evidenciou a baixa frequência do registro do peso da gestante ${ }^{(6)}$. Tendo em vista a importância da vigilância do estado nutricional durante o pré-natal, é necessário que os profissionais responsabilizem-se pela avaliação e orientação da gestante em relação ao seu estado nutricional, assistindo-a de forma integral ${ }^{(7)}$.

O mesmo deve ocorrer em relação ao período pós-parto. Apesar disso, no âmbito do Sistema Único de Saúde (SUS), ações das equipes de saúde no puerpério são destinadas quase que exclusivamente aos cuidados com o bebê, mediante consultas de puericultura ${ }^{(8)}$. Para a mulher, é prevista apenas uma consulta no final dos 40 dias após o parto ${ }^{(5)}$. Contudo, no período pós-parto, espera-se que haja um retorno ao peso 
pré-gestacional, e a principal intervenção de enfermagem para que este processo ocorra naturalmente é o incentivo à amamentação, pois a lactação, além dos benefícios para o bebê, pode ter efeito protetor contra a RPPP ${ }^{(2)}$.

Também vale ressaltar que a aceitação das mudanças corporais para atender as necessidades da maternidade nem sempre é um processo fácil, pois a pressão sobre as formas corporais da mulher prejudica a sua saúde mental. Além disso, ela necessita do suporte dos profissionais de saúde e também da família para a gestão positiva do corpo no puerpério ${ }^{(9)}$.

Estratégias para a assistência às puérperas são desafiadoras, pois, além de sobrecarga de atividades, mudança de papel, cansaço e preocupação em geral, elas também precisam cuidar de seus bebês, de modo a garantir-lhes saúde e bem-estar. Condutas voltadas para as mulheres nesse período geralmente não têm demanda espontânea. Por isso, ao gerenciar as ações sem considerar tais barreiras, as metas não são alcançadas. A atuação de profissionais de saúde no período puerperal, com o intuito de promover o retorno ao peso pré-gestacional, para impactar e diminuir os índices de obesidade em mulheres, exige conhecimento alinhado às suas necessidades sociais e de saúde nesse período de vulnerabilidade.

Ainda não existe consenso na literatura sobre os preditores da RPPP, pois os estudos apresentam conclusões discordantes. Deste modo, as revisões e metanálise indicam a necessidade de realização de mais pesquisas com o intuito de elucidar a temática ${ }^{(1,3-4)}$. As discordâncias, geralmente, estão relacionadas com as diferenças entre as populações estudadas - as características de cada grupo em estudo, assim como as variáveis sociodemográficas, culturais e econômicas selecionadas. Por isso, estudos regionais, que possibilitem verificar como este fenômeno comporta-se diante do perfil das mulheres são importantes. Diante disso, a principal questão desta pesquisa foi: Qual característica influenciou em maior RPPP, considerando uma população específica, como aquela que é atendida no sistema público de saúde? As principais hipóteses pré-estabelecidas pelos pesquisadores foram que a RPPP estaria relacionada ao tipo de parto e à amamentação exclusiva.

Diante do exposto, definiu-se como objetivo deste estudo analisar a retenção de peso pós-parto em mulheres assistidas no serviço público de saúde em um município do Sul do Brasil.

\section{Método}

Estudo de coorte realizado com 85 puérperas no município de Maringá (PR), seguindo as instruções do STROBE em seu desenvolvimento.

O município de Maringá é o terceiro mais populoso do estado do Paraná, sede da $15^{\underline{a}}$ Regional de Saúde, com estimativa populacional de 403 mil pessoas e 85\% de cobertura da Atenção Básica, que é composta, resumidamente, por 34 Unidades Básicas de Saúde (UBS) e 74 equipes de Estratégia de Saúde da Família (ESF). Em 2013, com a implantação do Programa Mãe Maringaense, um dos resultados alcançados foi a melhoria dos indicadores de acesso ao pré-natal, com aumento na proporção de nascidos vivos de mães com sete ou mais consultas, que atingiu $83,9 \%$ em $2016^{(10)}$.

No cálculo do tamanho amostral, considerou-se o número de nascimentos de residentes ocorridos no município no ano de 2015 (3.004). Dentre esses, os que foram realizados em serviço público (1.711 - 58,9\%). De posse deste total de nascimentos, considerou-se um nível de confiança de $95 \%$, erro de 3\%, e prevalência de 13\% de RPPP maior que $5 \mathrm{~kg}$ após 12 meses pós-parto ${ }^{(11)}$, acrescidos de $20 \%$ para possíveis perdas, o que resultou em uma amostra mínima de 462 puérperas.

Os critérios de inclusão previamente estabelecidos foram: puérperas com gestação única de conceptos vivos e idade gestacional igual ou superior a 37 semanas. Já os critérios de exclusão foram: não saber informar e nem ter registro no cartão da gestante do peso pré-gestacional e ao final da gestação. Por sua vez, considerou-se perda do seguimento/acompanhamento: mudança para outro município (26); manifestação verbal de desistência da participação (11); não 
localização da entrevistada nos telefones e endereços fornecidos por ela (154); incompatibilidade de horário para realização da visita domiciliar, após pelo menos cinco tentativas (96); e tempo do parto transcorrido inferior a seis meses ao término do período destinado à coleta de dados (90).

Os dados foram coletados no período de dezembro de 2017 a setembro de 2019, em dois momentos: no pós-parto imediato, depois de transcorridas 12 horas, e seis meses após, devido à importância do aleitamento materno exclusivo durante os primeiros seis meses e sua provável influência sobre a RPPP materna ${ }^{(12)}$.

Para a constituição do grupo a ser estudado, no período de dezembro de 2017 a setembro de 2019, foram realizadas buscas ativas diariamente nos hospitais em estudo até que o número previamente definido fosse alcançado. Nesse momento, foram consultados os prontuários para identificação das puérperas que atendiam aos critérios de inclusão e para coleta de informações, inclusive do cartão da gestante. Foi também verificado o peso corporal na balança eletrônica disponível no setor. A entrevista foi realizada com aquelas que aceitaram participar do estudo.

Seis meses após o parto, foi realizada visita domiciliar com entrevista, abordando hábitos de vida, aspectos emocionais e amamentação durante os primeiros seis meses de vida. Também verificou-se o peso corporal em balança portátil eletrônica. Ressalta-se que, devido às dificuldades para agendar as visitas domiciliares, 12 puérperas foram visitadas com atraso máximo de até 60 dias.

A variável dependente foi RPPP, utilizada tanto categórica (considerada presente quando maior que $1 \mathrm{~kg}$ ), quanto contínua (média e desvio padrão em $\mathrm{kg}$ ), definida pela diferença entre o peso pré-gestacional e o aferido na visita domiciliar.

As variáveis independentes e categóricas foram: características socioeconômicas (faixa etária; estado civil; trabalho remunerado e classificação econômica de acordo com a Associação Brasileira de Empresas e Pesquisa; obstétricas (multípara; cesariana anterior e tipo de parto atual); índice de massa corporal (IMC) em quatro momentos (peso/altura ${ }^{2}$ - peso antes da $1^{\underline{a}}$ gestação (peso recordado); peso pré-gestacional atual (aquele referido até dois meses antes da concepção ou descoberta da gestação, ou, em casos de a mulher não se recordar, considerou-se o peso anotado no cartão do pré-natal até a $14^{\underline{a}}$ semana de gestação); peso gestacional final (referido pela mulher, considerando mensuração feita pelo menos 30 dias antes da data do parto ou o peso anotado no cartão da gestante referente à última consulta de pré-natal, desde que tivesse ocorrido até 30 dias antes da data do parto); e peso corporal seis meses após o parto (aferido na visita domiciliar). Para classificação do estado nutricional com base nos valores do IMC, foi considerado excesso de peso, quando superior a $28,8 \mathrm{~kg} / \mathrm{m}^{2}$ no final da gestação ${ }^{(5)}$. Para os demais momentos, considerou-se o valor $24,9 \mathrm{~kg} / \mathrm{m}^{2}$.

O ganho de peso gestacional (diferença entre o peso no final da gestação e o peso pré-gestacional) foi considerado excessivo quando maior que: $18 \mathrm{~kg}$, para as mulheres que, antes da gestação, eram classificadas como abaixo do peso; $16 \mathrm{~kg}$, para as eutróficas; $11,5 \mathrm{~kg}$, para aquelas com sobrepeso; e $9 \mathrm{~kg}$, para as obesas ${ }^{(13)}$.

No tocante aos hábitos de vida, os alimentares foram investigados com base em três questões, sendo duas do Vigitel: frequência semanal de consumo de refrigerante/suco artificial adoçado e consumo semanal de produtos industrializados; e comer rápido. Quanto à prática de atividade física, investigou-se a presença de sedentarismo e relato de alguma atividade física (AF).

Quanto às características da amamentação, foi investigada a presença de aleitamento materno exclusivo (AME) aos seis meses de vida; introdução de fórmula láctea ou leite antes dos seis meses; e amamentação durante a noite.

Por fim, em relação aos aspectos emocionais seis meses após o parto, foram aplicados três instrumentos: Escala de estresse percebido, versão com 14 itens (PSS 14), cujo escore total varia de 0 a 56 pontos $^{(14)}$; Questionário de três fatores alimentares (TFEQ-R21) com três domínios: restrição cognitiva (RC), alimentação emocional (AE) e descontrole alimentar (DA). O escore total 
de cada domínio varia de 0 a 100 pontos $^{(15)}$; e Escala de figuras de Stunkard, constituída por nove desenhos femininos, nos quais cada figura é mais volumosa que a anterior. Ela é usada para medir a insatisfação corporal, tomando como ponto de partida a indicação do desenho no qual a pessoa se reconhece e a indicação daquele que gostaria de ter. A diferença entre as duas figuras aponta a intensidade da insatisfação corporal ${ }^{(16)}$.

A análise descritiva e estatística dos dados foi realizada no software SPSS $\AA$, considerando $\alpha=5 \%$. O p-valor foi determinado com o uso do teste Qui-quadrado. Também foram calculados as médias e o desvio padrão das variáveis contínuas e os percentuais para as variáveis categóricas.

O estudo foi conduzido de acordo com os padrões éticos nacionais para pesquisas com seres humanos. O projeto foi aprovado pelo Comitê de Ética em Pesquisa com Seres Humanos da instituição signatária (Certificado de Apresentação de Apreciação Ética n $n^{\circ} 70317817.3 .0000 .0104 / 2017$ e Parecer $\mathrm{n}^{\mathrm{o}}$ 2.180.586/2017). Todas as participantes assinaram o Termo de Consentimento Livre e Esclarecido (TCLE).

\section{Resultados}

Foram entrevistadas 85 mulheres após o parto. A média de tempo, em meses, para a visita domiciliar foi 7,1 $\pm 1,4$ meses. A RPPP maior que $1 \mathrm{~kg}$ esteve presente em 46 (54,1\%) entrevistadas; a maioria que reteve peso tinha 25 anos ou mais (62,5\%), com união estável ou casada $(55,1 \%)$, com trabalho remunerado (61,3\%), já havia sido submetida a cesariana anterior $(55,9 \%)$ e realizou cesariana novamente (56,6\%). A RPPP também apresentou associação estatisticamente significativa com estado nutricional eutrófico ou baixo peso no período pré-gestacional (65,8\%; $\mathrm{p}=0,05)$; com excesso de peso aos seis meses após o parto $(61,8 \% ; \mathrm{p}=0,05)$, e com ganho de peso gestacional excessivo $(68,4 \% ; \mathrm{p}=0,02)$ (Tabela 1$)$.

Tabela 1 - Perfil das mulheres segundo retenção de peso pós-parto após seis meses do parto. Maringá, Paraná, Brasil - 2019. (N=85)

(continua)

\begin{tabular}{|c|c|c|c|c|c|c|c|}
\hline \multirow{2}{*}{ Variáveis } & \multicolumn{7}{|c|}{ Retenção de peso pós-parto } \\
\hline & Sim & $\%$ & Não & $\%$ & Total & $\%$ & p-valor $(1)$ \\
\hline \multicolumn{8}{|l|}{ Faixa etária (em anos) } \\
\hline Menor que 24 & 26 & 49,1 & 27 & 50,9 & 53 & 62,4 & 0,23 \\
\hline 25 ou mais & 20 & 62,5 & 12 & 37,5 & 32 & 37,6 & \\
\hline \multicolumn{8}{|l|}{ Estado civil } \\
\hline Solteira/Viúva/Separada & 3 & 42,9 & 4 & 57,1 & 7 & 8,2 & 0,53 \\
\hline União estável/Casada & 43 & 55,1 & 35 & 44,9 & 78 & 91,8 & \\
\hline \multicolumn{8}{|l|}{ Trabalho remunerado } \\
\hline Sim & 19 & 61,3 & 12 & 38,7 & 31 & 36,5 & 0,32 \\
\hline Não & 27 & 50,0 & 27 & 50,0 & 54 & 63,5 & \\
\hline \multicolumn{8}{|l|}{ Classe econômica C,D,E(2) } \\
\hline Sim & 32 & 54,2 & 27 & 45,8 & 59 & 69,4 & 0,97 \\
\hline Não & 14 & 53,8 & 12 & 46,2 & 26 & 30,6 & \\
\hline \multicolumn{8}{|l|}{ Multípara } \\
\hline $\operatorname{Sim}$ & 27 & 54,0 & 23 & 46,0 & 50 & 58,8 & 0,98 \\
\hline Não & 19 & 54,3 & 16 & 45,7 & 35 & 41,2 & \\
\hline \multicolumn{8}{|l|}{ Cesariana anterior(3) } \\
\hline Sim & 19 & 55,9 & 15 & 44,1 & 34 & 68,0 & 0,70 \\
\hline Não & 8 & 50,0 & 8 & 50,0 & 16 & 32,0 & \\
\hline \multicolumn{8}{|l|}{ Parto atual } \\
\hline Vaginal & 16 & 50,0 & 16 & 50,0 & 32 & 37,6 & 0,55 \\
\hline Cesariana & 30 & 56,6 & 23 & 43,4 & 53 & 62,4 & \\
\hline \multicolumn{8}{|c|}{$\begin{array}{l}\text { Estado nutricional antes da primeira } \\
\text { gestação }\end{array}$} \\
\hline Excesso de peso & 11 & 55,0 & 9 & 45,0 & 20 & 23,5 & 0,93 \\
\hline Eutrófico/abaixo do peso & 35 & 53,8 & 30 & 46,2 & 65 & 76,5 & \\
\hline
\end{tabular}


Tabela 1 - Perfil das mulheres segundo retenção de peso pós-parto após seis meses do parto. Maringá, Paraná, Brasil - 2019. (N=85)

(conclusão)

\begin{tabular}{|c|c|c|c|c|c|c|c|}
\hline \multirow{2}{*}{ Variáveis } & \multicolumn{7}{|c|}{ Retenção de peso pós-parto } \\
\hline & Sim & $\%$ & Não & $\%$ & Total & $\%$ & p-valor(1) \\
\hline \multicolumn{8}{|c|}{ Estado nutricional pré-gestacional } \\
\hline Excesso de peso & 21 & 44,7 & 26 & 55,3 & 47 & 55,3 & 0,05 \\
\hline Eutrófico/abaixo do peso & 25 & 65,8 & 13 & 34,2 & 38 & 44,7 & \\
\hline \multicolumn{8}{|c|}{ Ganho de peso gestacional excessivo } \\
\hline Sim & 26 & 68,4 & 12 & 31,6 & 38 & 44,7 & 0,02 \\
\hline Não & 20 & 42,6 & 27 & 57,4 & 47 & 55,3 & \\
\hline \multicolumn{8}{|c|}{ Estado nutricional gestacional final } \\
\hline Excesso de peso & 29 & 52,7 & 26 & 47,3 & 55 & 64,7 & 0,73 \\
\hline Eutrófico/abaixo do peso & 17 & 56,7 & 13 & 43,3 & 30 & 35,3 & \\
\hline \multicolumn{8}{|c|}{$\begin{array}{l}\text { Estado nutricional após seis meses do } \\
\text { parto }\end{array}$} \\
\hline Excesso de peso & 34 & 61,8 & 21 & 38,2 & 55 & 64,7 & 0,05 \\
\hline Eutrófico/abaixo do peso & 12 & 40,0 & 18 & 60,0 & 30 & 35,3 & \\
\hline
\end{tabular}

Fonte: Elaboração própria.

(1) Teste Qui-quadrado.

(2) Renda média domiciliar menor que $\mathrm{R} \$ 2.705$, conforme classificação econômica da Associação Brasileira de Empresas e Pesquisa (ABEP).

(3) $n=50$.

Alguns hábitos e comportamentos durante o pós-parto não apresentaram associação estatisticamente significativa com RPPP. Entretanto, 30 $(60,0 \%)$ mulheres que introduziram fórmula ou leite de vaca antes dos seis meses e 21 (61,8\%) que não amamentavam durante a noite retiveram mais peso (Tabela 2).

Tabela 2 - Hábitos e comportamentos de mulheres segundo retenção de peso pós-parto após seis meses do parto. Maringá, Paraná, Brasil - 2019. (N=85)

(continua)

\begin{tabular}{|c|c|c|c|c|c|c|c|}
\hline \multirow{2}{*}{ Variáveis } & \multicolumn{7}{|c|}{ Retenção de peso pós-parto } \\
\hline & Sim & $\%$ & Não & $\%$ & Total & $\%$ & p-valor (1) \\
\hline \multicolumn{8}{|l|}{ Comer rápido } \\
\hline $\operatorname{Sim}$ & 25 & 58,1 & 18 & 41,9 & 43 & 50,6 & 0,45 \\
\hline Não & 21 & 50,0 & 21 & 50,0 & 42 & 49,4 & \\
\hline \multicolumn{8}{|c|}{$\begin{array}{l}\text { Frequência semanal de consumo } \\
\text { refrigerante/suco artificial adoçado }\end{array}$} \\
\hline $3 \mathrm{x}$ ou mais & 24 & 53,3 & 21 & 46,7 & 45 & 52,9 & 0,88 \\
\hline $2 \mathrm{x}$ ou menos & 22 & 55,0 & 18 & 45,0 & 40 & 47,1 & \\
\hline \multicolumn{8}{|c|}{$\begin{array}{l}\text { Frequência semanal de consumo } \\
\text { produtos industrializados }\end{array}$} \\
\hline $3 \mathrm{x}$ ou mais & 35 & 55,6 & 28 & 44,4 & 63 & 74,1 & 0,65 \\
\hline $2 \mathrm{x}$ ou menos & 11 & 50,0 & 11 & 50,0 & 22 & 25,9 & \\
\hline \multicolumn{8}{|l|}{ Sedentarismo } \\
\hline $\operatorname{Sim}$ & 35 & 51,5 & 33 & 48,5 & 68 & 80,0 & 0,33 \\
\hline Não & 11 & 64,7 & 6 & 35,3 & 17 & 20,0 & \\
\hline \multicolumn{8}{|l|}{ Atividade física } \\
\hline $\operatorname{Sim}$ & 4 & 57,1 & 3 & 42,9 & 7 & 8,2 & 0,87 \\
\hline Não & 42 & 53,8 & 36 & 46,2 & 78 & 91,8 & \\
\hline \multicolumn{8}{|c|}{ Aleitamento materno exclusivo } \\
\hline $\operatorname{Sim}$ & 9 & 47,4 & 10 & 52,6 & 19 & 22,4 & 0,50 \\
\hline Não & 37 & 56,1 & 29 & 43,9 & 66 & 77,6 & \\
\hline
\end{tabular}


Tabela 2 - Hábitos e comportamentos de mulheres segundo retenção de peso pós-parto após seis meses do parto. Maringá, Paraná, Brasil - 2019. (N=85)

\begin{tabular}{|c|c|c|c|c|c|c|c|}
\hline \multirow{2}{*}{ Variáveis } & \multicolumn{7}{|c|}{ Retenção de peso pós-parto } \\
\hline & Sim & $\%$ & Não & $\%$ & Total & $\%$ & p-valor $(1)$ \\
\hline \multicolumn{8}{|c|}{ Introdução fórmula ou leite } \\
\hline Sim & 30 & 60,0 & 20 & 40,0 & 50 & 58,8 & 0,19 \\
\hline Não & 16 & 45,7 & 19 & 54,3 & 35 & 41,2 & \\
\hline \multicolumn{8}{|c|}{ Amamentação durante noite } \\
\hline Sim & 25 & 49,0 & 26 & 51,0 & 51 & 60,0 & 0,25 \\
\hline Não & 21 & 61,8 & 13 & 38,2 & 34 & 40,0 & \\
\hline
\end{tabular}

Fonte: Elaboração própria.

(1) Teste Qui-quadrado.

Sobre a média dos valores antropométricos da amostra, observa-se que o IMC foi classificado como sobrepeso desde o período pré-gestacional até seis meses após o parto. A média do ganho de peso gestacional foi superior ao classificado como adequado para o IMC sobrepeso amostral pré-gestacional e a média de RPPP foi $2,2( \pm 6,5)$ (Tabela 3).

Tabela 3 - Média das variáveis antropométricas das mulheres. Maringá, Paraná, Brasil - 2019

\begin{tabular}{lc}
\hline Variáveis antropométricas & Média \pm desvio padrão \\
\hline Índice de massa corporal antes primeira gestação & $23,2 \pm 4,5 \mathrm{~kg} / \mathrm{m}^{2}$ \\
Índice de massa corporal pré-gestacional & $26,1 \pm 5,4 \mathrm{~kg} / \mathrm{m}^{2}$ \\
Índice de massa corporal gestacional final & $31,0 \pm 5,2 \mathrm{~kg} / \mathrm{m}^{2}$ \\
Índice de massa corporal pós-parto imediato & $29,7 \pm 5,2 \mathrm{~kg} / \mathrm{m}^{2}$ \\
Índice de massa corporal após seis meses & $27,0 \pm 5,6 \mathrm{~kg} / \mathrm{m}^{2}$ \\
Ganho de peso gestacional & $12,7 \pm 5,4 \mathrm{~kg}$ \\
Perda de peso pós-parto imediato & $3,4 \pm 2,2 \mathrm{~kg}$ \\
Retenção de peso pós-parto após seis meses & $2,2 \pm 6,5 \mathrm{~kg}$ \\
\hline
\end{tabular}

Fonte: Elaboração própria.

Quanto aos aspectos emocionais, não foi observada relação estatisticamente significativa com a RPPP na análise bivariada (dados não apresentados em tabelas), porém as médias dos escores obtidos foram maiores do que os valores médios propostos individualmente pelas escalas utilizadas (Tabela 4). Acrescenta-se que 70 $(82,4 \%)$ mulheres relataram insatisfação com seu corpo e $61(71,8 \%)$ desejavam um corpo mais magro do que o atual.

Tabela 4 - Média dos escores das escalas referentes aos aspectos emocionais das mulheres após seis meses do parto. Maringá, Paraná, Brasil - 2019

\begin{tabular}{lcc}
\hline Escores emocionais & Pontuação & Média \pm desvio padrão \\
\hline Estresse percebido & 0 a 56 & $29,6 \pm 5,3$ \\
Restrição cognitiva & 0 a 100 & $53,5 \pm 10,1$ \\
Alimentação emocional & 0 a 100 & $65,0 \pm 21,3$ \\
Descontrole alimentar & 0 a 100 & $57,7 \pm 11,7$ \\
\hline
\end{tabular}

Fonte: Elaboração própria.

\section{Discussão}

A média de RPPP das mulheres no estudo $(2,2 \mathrm{~kg})$ foi maior do que a encontrada em estudo de base populacional com usuárias de serviços públicos e privados, realizado em Botucatu (SP) ${ }^{(17)}$, seis meses após o parto $(1,8 \mathrm{~kg})$. As divergências entre as médias de RPPP nas pesquisas brasileiras estão relacionadas aos diferentes perfis amostrais analisados, 
impossibilitando comparações entre os valores encontrados $^{(17)}$.

Mais da metade das mulheres apresentaram RPPP. Além disso, de acordo com a média do IMC pré-gestacional que indicava sobrepeso, a média de ganho de peso, segundo os parâmetros do Institute of Medicine (IOM), que deveria ser entre 7 e $11,5 \mathrm{~kg}^{(13)}$, foi maior.

Sendo assim, o ganho de peso gestacional excessivo apresentou associação com RPPP, o que corrobora a literatura. Estudo de coorte mostrou que a retenção de peso foi maior em mulheres que apresentavam baixo peso antes da gravidez. Aquelas com GPG excessivo foram mais propensas a manter o peso pós-parto e mais propensas a reter pelo menos $5 \mathrm{~kg}$, em comparação com mulheres com GPG adequado ${ }^{(1)}$. Estudo com 4.102 mulheres do Sul do Brasil avaliou a relação entre GPG e RPPP e encontrou GPG médio de $12 \mathrm{~kg}$ e prevalência de GPG excessivo de 33,5\%. A média de RPPP superior a 3 meses após o parto foi de 2,3 kg e, após 12 meses, foi de $1,4 \mathrm{~kg}^{(18)}$.

Outro dado antropométrico associado à RPPP foi o estado nutricional eutrófico pré-gestacional. Entretanto, ainda não há consenso na literatura a respeito do IMC pré-gestacional ser preditor da $\operatorname{RPPP}^{(18)}$. Estudo realizado com mulheres afro-americanas não observou associação entre RPPP e IMC pré-gestacional ${ }^{(19)}$. Do mesmo modo, a coorte de nascimentos realizada no Maranhão/Brasil também identificou que a RPPP não possuía relação direta com IMC pré-gestacional, pois sofria influência de outros fatores de risco e de proteção, tais como GPG excessivo e amamentação ${ }^{(12)}$. Em Washington (EUA), foi realizada uma coorte que investigou a RPPP com base na diferença entre o peso no início da segunda gestação e o peso anterior à primeira gestação. Concluiu que mulheres com excesso de peso pré-gestacional tiveram menor probabilidade de retornar ao peso inicial, inclusive quando tiveram o GPG adequado ${ }^{(20)}$.

Ressalta-se que, embora no presente estudo não tenha sido observada associação significativa entre características da alimentação no período do pós-parto e a RPPP, a maioria das participantes tinha baixo poder econômico, relatou comer rápido e uso frequente de produtos industrializados. Este resultado corrobora conclusão de estudo no qual se constatou que as puérperas alimentavam-se conforme a disponibilidade e as condições financeiras, o que pode, muitas vezes, dificultar o consumo de alimentos frescos, como frutas, legumes, verduras e carnes $^{(21)}$. A demanda de cuidados com o bebê e a falta de tempo para o autocuidado ${ }^{(22)}$ também podem ser os motivos de escolhas alimentares mais rápidas, como os lanches e os produtos industrializados.

É importante lembrar que, de modo geral, o acompanhamento da equipe de saúde no puerpério prioriza o cuidado centrado no bebê $\hat{~}^{(8)}$, com pouca ou nenhuma ação voltada para o estado nutricional materno. A orientação alimentar, por exemplo, é direcionada, quase que exclusivamente, para o atendimento das necessidades do bebê $\hat{~}^{(21)}$, provavelmente em decorrência da amamentação.

As características da amamentação também não apresentaram associação significativa com a RPPP, entretanto, dentre as mulheres que retiveram peso, a maior parte não amamentou exclusivamente ao seio durante a noite e até o sexto mês de vida da criança. A relação entre amamentação e perda de peso ainda não é consenso na literatura, pois existe uma complexidade de fatores envolvidos ${ }^{(2)}$.

A coorte de nascimentos realizada no Maranhão apontou que, quanto maior a duração da amamentação, menor é a RPPP. Indicou também que o GPG excessivo prejudicava o início e a manutenção do aleitamento materno ${ }^{(12)}$. Destarte, as mulheres obesas apresentavam menor probabilidade de amamentar, com início tardio e menor duração da amamentação, devido a aspectos hormonais, como o aumento da progesterona e da leptina produzidas e/ou armazenadas no tecido adiposo, o que inibia a secreção de prolactina e influenciava na ejeção do leite $^{(2)}$. Outro estudo também constatou que a obesidade pré-gestacional e o GPG excessivo estavam associados a um risco aumentado de lactogênese retardada ${ }^{(23)}$. 
Contudo, metanálise que incluiu 11 estudos de cinco diferentes países (EUA, Brasil, França, Geórgia e Croácia) não identificou, a priori, relação entre aleitamento materno e perda de peso no pós-parto. Entretanto, quando presente, só foi observada com mais frequência em mulheres que continuaram a amamentação até 12 meses pós-parto. Este fato levou os autores a concluírem que as diferenças observadas nos resultados dos estudos podiam estar relacionadas com a intensidade e a duração da amamentação, com a população em estudo (fonte, tamanho, localização, perda ao seguimento), forma de avaliação do peso, da RPPP, da amamentação e dos métodos estatísticos utilizados. A dificuldade em comprovar o papel da amamentação na perda de peso pós-parto deve-se também ao efeito de vários preditores conhecidos, como GPG excessivo, peso pré-gestacional, prática de atividade física, estilo de vida ${ }^{(4)}$ e hábitos alimentares.

Em relação aos aspectos emocionais, embora não tenha sido evidenciada associação significativa com RPPP, os escores encontrados estavam acima do ponto de corte médio proposto individualmente pelas escalas utilizadas. Se considerarmos o perfil das mulheres estudadas, a maioria apresentava IMC elevado desde o período pré-gestacional até o pós-parto e quase metade delas teve GPG excessivo. Sendo assim, observa-se que, além das repercussões físicas, o excesso de peso também podia favorecer a ocorrência de tristeza, baixa autoestima, impactar na prática da amamentação e no vínculo mãe e filho, e ainda na imagem corporal negativa ${ }^{(24)}$.

$\mathrm{Na}$ presente amostra, a prevalência de insatisfação corporal foi elevada, pois a maioria desejava um corpo mais magro, apontando duas, três, quatro e até mais figuras anteriores à que se reconheciam no presente.

Revisão integrativa que incluiu estudos publicados entre 2014 e 2018, abordando transtornos psicológicos no período puerperal, concluiu que a preocupação e insatisfação com a imagem corporal provocavam alterações emocionais. Além disso, apontou que as mudanças e adaptações que ocorriam no âmbito familiar e domiciliar também potencializavam a vulnerabilidade da mulher no período puerperal ${ }^{(25)}$.

Em revisão de escopo com artigos publicados entre 2014 e 2019, abordando a gestão do corpo no puerpério, os autores ressaltaram o aumento da preocupação com o excesso de peso nesse período, o que acreditaram ser decorrente do culto ao corpo perfeito das sociedades ocidentais, provocando falsas expectativas quanto ao corpo utópico no pós-parto ${ }^{(9)}$. Destacaram ainda que o desejo de recuperar rapidamente o corpo pré-gravídico prejudicava a saúde mental e o bem-estar das mulheres. Neste sentido, relataram que, no Reino Unido, as equipes de saúde tentavam trabalhar essas questões, demonstrando que as alterações da gestação não eram negativas e faziam parte do processo da maternidade. Acreditavam na importância de os profissionais de saúde ajudarem as mulheres a conseguirem lidar com isso de forma mais natural. Com isso, diminuiria a pressão que elas sofriam no pós-parto, não somente a respeito do peso, como também sobre a ocorrência de estrias e flacidez ${ }^{(9)}$.

A insatisfação com a imagem corporal também pode prejudicar a amamentação. O desmame, por sua vez, pode prejudicar ou dificultar a perda de peso. Acredita-se que o desmame pode ser decorrente, entre outros fatores, da inquietação com o aspecto das mamas, da baixa autoestima, da intenção de restaurar o corpo anterior à gestação ${ }^{(9)} \mathrm{e}$, até mesmo, do desejo de tomar algum medicamento para ajudar no emagrecimento. Entretanto, com medo de prejudicar o bebê, a mulher preferia deixar de amamentá-lo.

Destaca-se que os resultados deste estudo mostraram que a equipe de saúde deveria estar atenta à identificação de mulheres com GPG excessivo e/ou excesso de peso pré-gestacional, para implementar estratégias que favorecessem a perda de peso sem sofrimento psíquico, de forma natural e fisiológica. Neste sentido, seria importante que todos apoiassem e encorajassem amamentação exclusiva até seis meses e a manutenção da amamentação até dois anos; incentivassem a prática de atividade física, conforme as condições socioeconômicas e a disponibilidade de tempo; acompanhassem e orientassem 
a escolha de alimentos adequados; fornecessem apoio emocional e promovessem a melhora da autoestima.

Por fim, é importante destacar que o cálculo do IMC é uma tecnologia efetiva para o monitoramento do estado nutricional da população e para direcionar as ações em saúde no âmbito do serviço público. É de fácil mensuração, simples e sem custo, podendo ser utilizado por todos os profissionais de saúde, em especial pela equipe de enfermagem, tanto na atenção primária da saúde, como no ambulatório, nos serviços de pronto atendimento e nas unidades de obstetrícia dos hospitais. Apesar disso, é importante ressaltar que muitas mulheres deixaram de ser incluídas no estudo por falta desta informação no cartão da gestante, seja em relação ao início, seja em relação ao final da gestação.

Uma das limitações do estudo foi a perda elevada de participantes após a primeira etapa, decorrente de problemas operacionais por parte das pesquisadoras, tais como incompatibilidade de horário, indisponibilidade da puérpera para agendamento da visita domiciliar, ausência de auxílio financeiro para os deslocamentos, tempo gasto nos deslocamentos, devido à distância entre as residências, além de restrição na disponibilidade do veículo de uso familiar. Contudo, é importante destacar que o perfil das mulheres entrevistadas seis meses após o parto foi similar ao daquelas entrevistadas no puerpério imediato, o que minimiza o viés de seleção das mulheres acompanhadas durante o período da pesquisa. Outra limitação da pesquisa foi trabalhar com IMC ou estado nutricional, utilizando a relação do peso com a altura, mas não mensurar a taxa e a distribuição da gordura no organismo. De qualquer modo, a generalização dos resultados é válida, pois o perfil das mulheres em estudo correspondeu ao de grande parte da população. Além disso, mesmo que a assistência à saúde seja diversa nas diferentes regiões brasileiras, muitos estudos apontam iguais preocupações com a atenção puerperal.

O estudo traz contribuições relevantes para a área da saúde da mulher, visto que a investigação dos preditores da RPPP em mulheres que tiveram o acompanhamento pré-natal e o parto pelo serviço público de saúde fortalece as políticas públicas direcionadas a esta população. Ademais, fundamenta a ação do enfermeiro, que é atuante em todas as esferas de atendimento à mulher no ciclo gravídico-puerperal.

O estudo tem importância para a enfermagem, em razão de apontar fragilidades na assistência ao puerpério, sobretudo pelo fato de ser centrada basicamente no bebê. Além disso, indica a necessidade de, no planejamento da assistência, os enfermeiros considerarem as dificuldades experienciadas pelas mulheres para retorno ao peso pré-gestacional e os fatores implicados nesta questão. Isto, certamente, ampliará a possibilidade de uma assistência mais ampliada e próxima das reais necessidades e focada na integralidade.

\section{Conclusão}

Neste estudo, os fatores associados significativamente à RPPP - estado nutricional pré-gestacional eutrófico ou abaixo do peso e o GPG excessivo - resultaram em um estado nutricional pós-parto imediato com excesso de peso, que permaneceu após seis meses do parto.

Mesmo a média da retenção de peso não sendo elevada, a perda de peso nos seis meses pós-parto não foi capaz de reduzir a média do IMC da amostra a ponto de se equiparar à média do IMC pré-gestacional. Este fato indica a importância do monitoramento do GPG durante as consultas de pré-natal. A amamentação, mesmo sem significância estatística com a RPPP neste estudo, deve ser incentivada pela equipe de saúde, que deve atentar para o fato de que seu papel é maior do que apenas nutrir o lactente. Os aspectos emocionais no período puerperal demonstraram a necessidade de acolhimento tanto por parte dos familiares e da rede de apoio como das ações das equipes da Atenção Primária à Saúde.

\section{Colaborações:}

1 - concepção, projeto, análise e interpretação dos dados: Lorenna Viccentine Coutinho Monteschio e Sonia Silva Marcon; 
2 - redação do artigo e revisão crítica relevante do conteúdo intelectual: Lorenna Viccentine Coutinho Monteschio, Sonia Silva Marcon, Evelin Matilde Arcain Nass, Cátia Campaner Ferrari Bernardy, Áurea Christina de Paula Corrêa, Patrícia Chatalov Ferreira e Débora Cristina Arruda;

3 - aprovação final da versão a ser publicada: Lorenna Viccentine Coutinho Monteschio, Sonia Silva Marcon, Evelin Matilde Arcain Nass, Cátia Campaner Ferrari Bernardy, Áurea Christina de Paula Corrêa, Patrícia Chatalov Ferreira e Débora Cristina Arruda.

\section{Referências}

1. Ha AVV, Zhao Y, Pham NM, Nguyen CL, Nguyen PTH, Chu TK, et al. Postpartum weight retention in relation to gestational weight gain and pre-pregnancy body mass index: A prospective cohort study in Vietnam. Obes Res Clin Pract. 2019;13(2):143-9. DOI: 10.1016/ j.orcp.2019.02.001

2. Falivene MA, Orden AB. Fatores do comportamento materno que influenciam a retenção de peso pós-parto. Implicações clínico-metabólicas. Rev Bras Saúde Mater Infant. 2017;17(2):251-9. DOI: 10.1590/1806-9304 2017000200003

3. Nogueira JL, Saunders C, Leal MC. Métodos antropométricos utilizados na avaliação da retenção do peso no período pós-parto: uma revisão sistemática. Ciênc saúde coletiva. 2015;20(2):407-20. DOI: 10.1590/1413-812320152 02.08112013

4. He X, Zhu M, Hu C, Tao X, Li Y, Wang Q, et al. Breast-feeding and postpartum weight retention: A systematic review and meta-analysis. Public Health Nutr. 2015;18(18):3308-16. DOI: 10.1017/S1368980015000828

5. Brasil. Ministério da Saúde. Secretaria de Atenção à Saúde. Departamento de Atenção Básica. Atenção ao pré-natal de baixo risco [Internet]. Brasília (DF); 2012 [cited 2020 Jun 10]. Available from: http:// bvsms.saude.gov.br/bvs/publicacoes/cadernos_ atencao_basica_32_prenatal.pdf

6. Vieira VCL, Barreto MS, Fernandes C, Scochi MJ. Análise da assistência pré-natal em municípios de diferentes portes populacionais do Paraná. Cienc
Cuid Saude. 2016;15(1):125-32. DOI: 10.4025/ cienccuidsaude.v15i1.16535

7. Ismail LC, Bishop DC, Pang RO, Ohuma EC, Kac GE, Abrams BT, et al. Gestational Weight Gain Standards Based on Women Enrolled in the Fetal Growth Longitudinal Study of the INTERGROWTH-21st Project: A Prospective Longitudinal Cohort Study. BMJ. 2016;71(7):383-5. DOI: $10.1136 / \mathrm{bmj} .1555$

8. Baratieri $\mathrm{T}$, Natal S. Ações do programa de puerpério na atenção primária: uma revisão integrativa. Ciênc saúde coletiva. 2019;24(11):422738. DOI: 10.1590/1413-812320182411.28112017

9. Fialho P, Antunes V, Madeira C, Amendoeira J. Promoção da capacidade da mulher para gerir o corpo no puerpério: uma scoping review. Rev UIIPS. 2020;8(1):223-37. DOI: 10.25746/ruiips. v8.i1.19894

10. Maringá. Secretaria Municipal de Maringá. Plano municipal de saúde de Maringá-PR 2018/2021 [Internet]. Maringá (PR); 2017 [cited 2021 Jan 4]. Available from: http://www2.maringa.pr.gov.br/ sistema/arquivos/02eda74d2112.pdf

11. Rode L, Kjærgaard H, Ottensen B, Damm P, Hegaard HK. Association between gestational weight gain according to body mass index and postpartum weight in a large cohort of Danish women. Matern Child Health J. 2012;16(2):406-13. DOI: $10.1007 /$ s10995-011-0775-Z

12. Chagas DC, Silva AAM, Ribeiro CCC, Batista RFL, Alves MTSSB. Efeitos do ganho de peso gestacional e do aleitamento materno na retenção de peso pós-parto em mulheres da coorte BRISA. Cad Saúde Pública. 2017;33(5): e00007916. DOI: 10.1590/0102-311x00007916

13. Institute of Medicine (US), National Research Council (US), Committee to Reexamine IOM Pregnancy Weight Guidelines, Rasmussen KM, Yaktine AL, editors. Weight Gain During Pregnancy: Reexamining the Guidelines [Internet]. Washington (DC): National Academies Press (US); 2009 [cited 2019 Aug 5]. Available from: http://www. ncbi.nlm.nih.gov/books/NBK32799/\#summary.s1

14. Luft CDB, Sanches SO, Mazo GZ, Andrade A. Versão brasileira da Escala de Estresse Percebido: tradução e validação para idosos. Rev Saúde Pública. 2007;41(127):606-15. DOI: 10.1590/ S0034-89102007000400015

15. Natacci LC, Ferreira Junior M. The three factor eating questionnaire - R21: tradução para o 
português e aplicação em mulheres brasileiras. Rev Nutr. 2011;24(3):383-94. DOI: 10.1590/S141552732011000300002

16. Scagliusi FB, Alvarenga M, Polacow VO, Cordas TA, Queiroz GKO, Coelho D, et al. Concurrent and discriminant validity of the Stunkard`s figure rating scale adapted into Portuguese. Appetite. 2006;47(1):77-82. DOI: 10.1016/j.appet.2006.02.010

17. Alves MS, Almeida MAM, Gomes CB, Ferrari AP, Parada CMGL, Carvalhaes MABL. Maior duração do aleitamento materno exclusivo reduz retenção de peso materno: resultados do estudo CLaB. Rev Bras Saúde Mater Infant. 2020;20(1):273-84. DOI: 10.1590/1806-93042020000100015

18. Flores TR, Nunes BP, Miranda VIA, Silveira MF, Domingues MR, Bertoldi AD. Ganho de peso gestacional e retenção de peso no pós-parto: dados da coorte de nascimentos de 2015, Pelotas, Rio Grande do Sul, Brasil. Cad Saúde Pública. 2020;36(11):e00203619. DOI: https://doi. org/10.1590/0102-311x00203619

19. Schneider CR, Biggio JR, Chandler-Laney PC. Association of early pregnancy body mass index with post-partum weight change among AfricanAmerican women. Clin Obes. 2018;8(3):170-5. DOI: doi.org/10.1111/cob.12241

20. Ketterl T, Dundas N, Roncaioli S, Littman A, Phipps A. Association of Pre-pregnancy BMI and Postpartum Weight Retention Before Second Pregnancy, Washington State, 2003-2013. Matern Child Health J. 2018;22(9):1339-44. DOI: 10.1007/ s10995-018-2514-1
21. Moreira LV, Barros DC, Baião MR, Cunha MB. "Quando tem como comer, a gente come": fontes de informações sobre alimentação na gestação e as escolhas alimentares. Physis. 2018;28(3):e280321. DOI: 10.1590/S0103-7331201 8280321

22. Lelis BDB, Pereira RC, Silva LFI, Leite AM, Dusso MIS, Bernardes NB. Acolhimento puerperal no contexto atribuído às primíparas. Id on Line Rev Mult Psic. 2019;13(45):287-301. DOI: 10.14295/idonline.v13i45.1702

23. Preusting I, Brumley J, Odibo L, Spatz DL, Louis JM. Obesity as a predictor of delayed lactogenesis II. J Hum Lact. 2017;33(4):684-91. DOI: $10.1177 / 0890334417727716$

24. Bergmeier H, Hill B, Haycraft E, Blewitt C, Lim S, Meyer C, et al. Maternal body dissatisfaction in pregnancy, postpartum and early parenting: An overlooked factor implicated in maternal and childhood obesity risk. Appetite. 2020;147:104525. DOI: 10.1016/j.appet.2019.104525

25. Frota CA, Batista CA, Pereira RIN, Carvalho APC, Cavalcante GLF, Lima SVA, et al. A transição emocional materna no período puerperal associada aos transtornos psicológicos como a depressão pós-parto. REAS. 2020;(48):e3237. DOI: $10.25248 /$ reas.e3237.2020

Recebido: 8 de janeiro de 2021

Aprovado: 18 de maio de 2021

Publicado: 4 de agosto de 2021

A Revista Baiana de Enfermagem utiliza a Licença Creative Commons - Atribuição-NãoComercial 4.0 Internacional. https://creativecommons.org/licenses/by-nc/4.0/

Este artigo é de acesso aberto distribuído sob os termos da Licença Creative Commons (CC BY-NC).

Esta licença permite que outros remixem, adaptem e criem a partir do seu trabalho para fins não comerciais. Embora os novos trabalhos tenham de lhe atribuir o devido crédito e não possam ser usados para fins comerciais, os usuários não têm de licenciar esses trabalhos derivados sob os mesmos termos. 